\title{
Les Plaintes de la Déploration de Florimond Robertet ou les apories de la poésie funèbre chez Marot
}

La Déploration de Florimond Robertet ${ }^{1}$ est un vaste poème composé en l'honneur du trésorier de François $I^{\text {er }}$, mort en 1527, alors que Clément Marot est à la cour du roi depuis un an seulement. Jusqu'à présent, le poète s'était illustré essentiellement par des pièces de facture et de ton plus modestes: épitaphes, ballades, rondeaux ou chansons, regroupés dans une Adolescence clémentine à laquelle la Déploration de Florimond Robertet semble mettre fin, puisqu'elle figure en tête des " autres oeuvres [...] faictes depuis leage de son Adolescence » dans le premier recueil édité par Marot en 1532. Or cette déploration funèbre est un morceau de bravoure de la poésie de cour, issue d'une longue tradition et possédant, de fait, de nombreux attendus rhétoriques ${ }^{2}$ parmi lesquels figurent une lamentation, l'éventuel récit des circonstances du décès, l'éloge du mort puis une consolation, qui passe souvent par la survie du défunt par la gloire et sa canonisation triomphale - le tout dans un cadre allégorique, où différentes personnifications s'expriment. De ce point de vue, la Déploration de Florimond Robertet se présente de prime abord de façon très conventionnelle, reconduisant les pratiques poétiques des prédécesseurs de Marot.

En effet, après un prologue dans lequel l'auteur se plaint de devoir parler d'une mort alors que sa plume est d'ordinaire enjouée - d'une façon qui n'est pas sans rappeler le début de la Couronne Margaritique de Lemaire ${ }^{3}$ - Marot raconte comment il a croisé un cortège funèbre à Torfou, encadré par des personnifications telles que «Dame Rommaine», "Republique Françoyse» et «la Mort». Comprenant qu'il s'agit de la procession funèbre de Robertet, il effectue une lamentation reposant sur un éloge des qualités professionnelles et personnelles du défunt - la célébration de l’homme de lettres en plus du serviteur zélé étant un autre lieu commun du genre, notamment illustré par Cretin dans sa Plainte sur le trespas de Byssipat'. Ensuite, Republique Françoyse et la Mort s'expriment tour à tour, selon une structure rappelant la Complaincte et epytaphe du feu roy Charles d'Octovien de Saint-Gelais ${ }^{5}$. Le discours de Republique Françoyse est une triple plainte portant sur la perte d'un serviteur particulièrement compétent (en termes rhétoriques, cela correspondrait à la lamentation), dénonçant l'action vile de la Mort (invective), lui reprochant de plonger les proches de Robertet et le peuple dans une si profonde tristesse (accusation de malice). Si ces plaintes sont topiques dans la déploration funèbre, il est pourtant rare que la Mort y réponde, et même surprenant que son discours occupe la

\footnotetext{
${ }^{1}$ Clément Marot, Euvres poétiques, éd. G. Defaux, t. 1, Paris, Bordas (« Classiques Garnier »), 1990, p. 207-223. Toutes nos citations renverront à cette édition.

${ }^{2} \mathrm{Il}$ n'existe pas de traité de rhétorique abordant directement la déploration funèbre aux $\mathrm{XV}^{\mathrm{e}}$ et $\mathrm{XVI}{ }^{\mathrm{e}}$ siècles : les poètes, nourris de l'enseignement rhétorique, ont à leur disposition les traités de l'Antiquité, mais qui se bornent dans l'ensemble aux grands genres de discours (démonstratif, délibératif et judiciaire). Font exception, pour l'oraison funèbre, les traités de Ménandre et du pseudo-Denys. Les arts de seconde rhétorique, qui fleurissent à la fin du Moyen Âge, se concentrent sur les formes poétiques et la versification, de même que les arts poétiques qui leur succèdent. Plus utiles, et datant du début du $\mathrm{XVI}$ e siècle, sont les traités épistolaires : le classement des différents types de lettres, dans Le Grant et vray art de pleine rhetorique de Fabri ou le De Conscribendis epistolis d'Érasme par exemple, croise en effet précisément celui des types de discours. Mais ces traités se situent dans le cadre d'une «conversation entre absents » qui, même dans un contexte hiérarchique marqué, ne saurait entièrement rendre compte de la rhétorique d'un poète s'adressant par devoir à un monarque et par extension à toute une cour ou une nation. Si la déploration funèbre n'est donc guère théorisée, les nombreux exemples des Rhétoriqueurs présentent une structure offrant peu de variations : leur étude permet d'établir un canon à l'intérieur duquel les poètes développent une pensée et une esthétique propres. Voir ainsi Claude Thiry, La Plainte funèbre, Brepols, Turnhout-Belgium, 1978 ; Christine Martineau-Génieys, Le thème de la Mort, dans la poésie française de 1450 à 1550, Paris, Champion, 1978 et Claude Blum, La Représentation de la mort dans la littérature de la Renaissance, Paris/Genève, Honoré Champion/Slatkine, 1989, t. 1, p. 157-291.

3 Jean Lemaire de Belges, Euvres, Jean-Auguste Stecher (éd.), [Louvain, 1882-1885], Genève, Slatkine Reprints, 1969 , vol. IV, p. 15.

${ }^{4}$ Guillaume Cretin, Euvres poétiques, éd. Kathleen Chesney, Genève, Slatkine Reprints, 1977 [Paris, 1932], p. 73-93.

${ }^{5}$ Octovien de Saint-Gelais et André de La Vigne, Le Vergier d'Honneur, Paris, Jean Petit, circa 1512, f. $1 \mathrm{IV} \mathrm{v}^{\circ}-\mathrm{mII} \mathrm{v}^{\circ}$.
} 
place de la consolation glorieuse et profane attendue, pour lui substituer un prêche évangélique supposé effacer les pleurs et la crainte de l'assistance. Or, dans sa réponse, la Mort se plaint à son tour : d'une part d'être ainsi accusée (elle formule ainsi une défense), d'autre part de l'incohérence des hommes qui se plaignent d'elle mais font des rituels très bénéfiques (c'est une nouvelle accusation, assortie d'une critique anti-papale), et enfin de la bêtise, et même de l'hérésie des hommes qui ont oublié que seule la mort permet de quitter un monde de péché et d'accéder au salut, grâce au sacrifice du Christ. La critique porte peut-être cette fois aussi sur la poésie courtisane allégorique et " païenne ». Des études ont ainsi interprété la Déploration de Florimond Robertet comme un manifeste évangélique, dans lequel Marot détournerait, pour des raisons idéologiques, les déplorations de ses prédécesseurs et exhiberait la vanité voire l'hypocrisie des plaintes funèbres traditionnelles, telles qu'elles sont formulées par Republique Françoyse ${ }^{6}$. Pour terminer, le poète décrit la réaction du peuple : malgré ce long discours, tout le monde continue de plaindre Robertet et de craindre la Mort comme dans les premiers vers.

Les plaintes se succèdent donc sans recevoir de réponse - l'interlocuteur suivant se contentant de formuler à nouveau sa propre plainte selon ses propres intérêts. Alors que, chez les Rhétoriqueurs, la déploration funèbre doit consoler de la perte subie et souder la communauté autour de valeurs réaffirmées, il semble au contraire que, dans la Déploration de Florimond Robertet, ces multiples plaintes peinent à créer du lien et exhibent même différentes sortes de fractures portant atteinte au genre même de la déploration funèbre. Loin de soulager les sujets endeuillés, de réaliser une plénitude dans l'expression de la douleur et de mener, grâce à une réception convenue, vers une consolation, ce foisonnement de plaintes variées voire contradictoires renvoie dos à dos des représentations éthiques, politiques, poétiques ou religieuses permettant traditionnellement de donner sens à la mort. Comment comprendre ce piétinement de la déploration? En quoi ces plaintes révèlent-elles des tensions et apories portant aussi bien sur la tradition de la déploration funèbre des Rhétoriqueurs que sur le message évangélique diffusé ?

Nous verrons que ces plaintes successives accusent une menace de déliaison provoquée par la mort, entre les différentes entités sociales et religieuses, mais aussi entre les différentes facettes de Marot et de l'humaine condition - sujet, ami, croyant, poète - dont on étudiera tour à tour les oppositions.

\section{I- Tension éthique, entre l’ami et le courtisan}

La Déploration de Florimond Robertet célèbre un personnage particulièrement éminent du royaume de France, serviteur illustre de la couronne depuis Charles VIII ${ }^{7}$. Fils du poète Jean Robertet, Florimond était aussi un homme de lettres, mécène, à l'écoute des idées évangéliques. Non seulement Marot perd un très illustre serviteur de la cour où il vient d'arriver, mais aussi un défenseur de la cause évangélique et un ami des Muses comme lui. Tous ces éléments justifient la composition d'une œuvre d'ampleur lui rendant dignement hommage, par laquelle le poète peut aussi faire ses preuves dans cette cour où il a récemment succédé à son père comme poète du roi. Dès le début de sa Déploration de Florimond Robertet, le poète explique en des vers très émouvants qu'il choisit de «tremper [sa plume] en ancre d'amertume » (v. 6) moins par obligation liée au service de François I ${ }^{\text {er }}$ que par amitié pour le défunt :

Ainsi le fault, \& quand ne le fauldroit,

Mon cueur (helas) encores le vouldroit :

\footnotetext{
"Voir notamment Mario Richter, "L’evangelismo di Clément Marot, Lettura della Déploration de Florimont Robertet», Bibliothèque d'Humanisme et Renaissance, 35-2, 1973, p. 247-258, Martineau-Génieys, Le thème de la Mort, op. cit., p. $439-483$ et Blum, La Représentation de la mort dans la littérature de la Renaissance, op. cit., p. 160-193.

${ }^{7}$ «Ce fut la Plume en sage main baillée, / Qui ne fut oncq (comme je croy) taillée / Que pour servir en leurs secretz les Roys : / Aussi de reng elle en a servy troys » (v. 141-144).
} 
Et quand mon cueur ne le vouldroit encores,

Outre son vueil contrainct y seroit ores

Par l'aiguillon d'une mort, qui le poinct :

Que dis je mort? D'une mort n'est ce point :

Ains d'une amour : car quand chascun mourroit

Sans vraye Amour, plaindre on ne le pourroit :

Mais quand la Mort a faict son malefice,

Amour adonc use de son office,

Faisant porter aux vrays Amys le dueil [...]. (v. 7-17)

Marot se défend de rendre hommage à Robertet en raison de l'éminence de sa position à la cour de François $I^{\text {er }}$ et souligne au contraire la «vraye amour » portée au défunt. L'idée n'est pas nouvelle ${ }^{8}$, mais Marot développe particulièrement sa plainte personnelle. Il l'étaye plus loin en précisant que si le roi a ordonné une si grande pompe funèbre pour Robertet, c'est moins par convention que comme témoignage de reconnaissance, et même « d'amour », pour un service dévoué :

Monstrant au doid, combien d'amour desservent

De leurs Seigneurs les Servans, qui bien servent. (v. 111-112)

L'attachement personnel de Marot à Robertet s’inscrit aussi dans le goût partagé pour la poésie. Dans son éloge, le poète souligne les qualités littéraires du trésorier :

C'est celle Plume, où modernes espritz

(Soubz ses patrons) leur sçavoir ont appris [...]. (v. 139-140)

Là encore, cet éloge n'est guère original dans le corpus des déplorations des Rhétoriqueurs, mais Marot lui confère une dimension particulière en rappelant que Robertet l'avait protégé (ainsi que son père') et avait sans doute favorisé son entrée au service du roi. Il est ainsi

Celluy, qui fut la toute ronde Sphere,

Par où guettoys ma fortune prospere. (v. 163-164)

Dès le début du poème, alors même (ou parce) que Marot s'essaye pour la première fois au grand genre de la déploration funèbre, il semble congédier les motifs courtisans de la plainte ${ }^{10}$ pour souligner de façon très précise combien son amitié pour le défunt justifie une « si piteuse harangue » (v. 158).

Pourtant, une déploration courtisane succède à cette première plainte, formulée par Republique Françoyse. L'allégorie du pouvoir énonce en effet une plainte typique de la Grande Rhétorique, faisant l'éloge du trépassé, s'adressant tour à tour aux vivants et invectivant la Mort. La louange de Robertet insiste cette fois sur ses qualités de « bon Serf » (v. 193), « diligent» (v. 251) à la tâche. La description de l'assemblée en deuil sert une comparaison épique entre Hector et le trésorier soulignant ses compétences et son dévouement pour le royaume ${ }^{11}$. Les éloges suivants sont formulés par des synecdoques :

\footnotetext{
${ }^{8}$ Elle est notamment illustrée dans la Plainte sur le trespas de Byssipat de Cretin, qui date de 1512.

${ }_{9}^{9}$ C'est ce que confirme la «Ballade de Maistre Jehan Marot presentée à Monsieur le Tresorier Robertet » (Les Deux Recueils, éd. Gérard Defaux et Thierry Mantovani, Genève, Droz, 1999, p. 195-196).

${ }^{10}$ Ce traitement personnel de la déploration funèbre apparait déjà dans sa modeste «Complaincte d'une Niepce, sur la mort de sa Tante », composée entre 1524 et 1526 (CEuvres poétiques, op. cit., p. 97-99).

11 "Quiconques fut Hector aux armes né, / Robertet fut nostre Hector en sagesse » (v. 202-203). Au XVI e siècle, la croyance dans les origines troyennes de la France était répandue et a longuement été développée dans les Illustrations de Lemaire : faire 
Tu as froissé la main tant imitable,

Qui au proffit de moy lasse [malheureuse] escripvoit :

Tu as cousu la bouche veritable :

Tu as percé le cueur tant charitable,

Et assommé le Chef, qui tant sçavoit. (v. 221-225)

Cet encomium de la triste Republique est d'autant plus efficace qu'il vient en contrepoint d'un autre discours épidictique, celui du blâme de la Mort, qualifiée de « vieille effacée, infecte, image immunde, / craincte de gens, pensement soucieux » (v. 213-214). L’invective contre la Mort, la lamentation partagée avec le peuple accrue par la mesure de la perte d'un serviteur si compétent sont autant de plaintes attendues dans une déploration courtisane.

Mais ici, elles semblent d'autant plus prévisibles qu'elles répètent la «si piteuse harangue »du poète. Ainsi Marot a-t-il commencé par décrire de façon dépréciative «la Mort hydeuse » et son char (v. 43-54), préparant l'invective de Republique Françoyse. Puis il a composé un éloge du défunt, serviteur royal zélé autant qu'écrivain de talent, à travers un développement sur l'aile du blason de Robertet. La description de ce blason ainsi que des qualités de serviteur et de protecteur de son possesseur ont la forme d'une énigme au terme de laquelle est enfin dévoilé le nom du défunt, que le poème évoque déjà depuis près de 150 vers :

O vous humains, qui escoutez ma plaincte,

Qui est celluy, qui eut ceste Esle paincte

En son escu? Vous en faut-il doubter?

Sentez vous point, quand venez à gouster

Ce, que je dy en mon triste mottet,

Que c'est le bon Florimond Robertet?

En est-il d'aultre en la vie mortelle,

Pour qui je disse une louange telle ? (v. 147-154)

Cet effet de retard de l'annonce du nom du défunt, qui accroît le pathos du poème tout en portant à l'éloge à son comble, est un topos des déplorations funèbres et se retrouve notamment dans chacune de celles de Cretin. Mais de commun, ce lieu devient redondant sous la plume de Marot qui le réutilise au terme du discours de Republique Françoyse :

Si son nom propre à dire on me semond,

Je respondray, qu'à son los se compasse :

Son los fleurit, son nom est Florimond,

Ung Mont flory, ung plus que flory Mont [...]. (v. 253-256)

La fin élogieuse du retard du nom ainsi que la surenchère encomiastique dans la rime équivoquée ne font que répéter le discours encadrant. Cela révèle combien les plaintes de l'ami et du courtisan, pour semblables qu'elles puissent être, ne se superposent guère. Ainsi prise à défaut, la plainte de Republique Françoyse trahit une fracture du sujet avec l'ami : même si les deux ont recours aux mêmes lieux communs pour composer leur plainte, leur redondance révèle précisément qu'ils ne s'écoutent pas, ne communiquent pas. Non prise en compte par l'auditoire, la plainte est vouée à se répéter et à perdre sa

du serviteur de la couronne un équivalent du fils de Priam, illustre roi de Troie, est sans doute le plus haut titre honorifique que peut décerner Republique Françoyse. 
substance : son pathos réside donc moins dans le choix des mots et des lieux que dans le lien qu'elle est ou non capable d'établir en fonction de sa situation d'énonciation.

Cette répétition sur fond de tension éthique affecte en outre l'écriture même de la déploration, qui piétine et peine à se sortir des lieux communs éculés des Rhétoriqueurs. D’éthiques, les enjeux des plaintes de la Déploration de Florimond Robertet se font rhétoriques et poétiques et marquent là aussi un boitement de la composition, qui peine à définir sa nature même.

\section{II- Tension poétique et rhétorique, entre le poète de cour et le poète évangélique}

Alors que le canon de la poésie de cour exigerait une consolation triomphale après les lamentations et éloges de Robertet, la Mort poursuit les plaintes de Republique Françoyse et les retourne même contre elle, en condamnant le manque de foi des hommes que son discours reflète. La critique a unanimement entendu dans ce discours la voix de Marot qui formulerait directement, voire de façon provocante à travers le choix de ce locuteur surprenant, un véritable sermon évangélique. Or ce manifeste d'une pensée et d'une foi renouvelées, selon Martineau-Génieys ou Blum ${ }^{12}$, ne peut s'énoncer dans le cadre conventionnel et profane des déplorations des Rhétoriqueurs, d'où l'enchaînement des plaintes (sous forme de lamentation, invective, sermon ou silence) au lieu d'une consolation triomphale, allégorique et profane comme il pouvait s'en trouver chez Saint-Gelais ou Lemaire. L'évangélisme de Marot détourne les attendus d'une déploration funèbre qui commençait pourtant bien dans la tradition : les critiques y ont vu une condamnation de ce genre, ainsi qu'une liquidation. Nous ne pensons toutefois pas que le geste de Marot soit aussi subversif à l'égard de la tradition poétique, pour plusieurs raisons. D'une part, en 1527, François I ${ }^{\text {er }}$ est réceptif aux idées évangéliques, de même que son trésorier Robertet: le discours de la Mort dans la Déploration de Florimond Robertet relève donc en partie des circonstances et est aussi un hommage au défunt. D'autre part, Marot tout juste arrivé à la cour souhaite sans doute moins renverser toute une tradition que montrer sa connaissance des attendus du genre et la subtilité de son traitement. Il écrira d'ailleurs quelques années plus tard des déplorations funèbres directement inspirées de Lemaire ou de Cretin, telles que l'Eglogue sur le trespas de ma dame Loyse de Savoye, célébrant la mère du roi, ou la Complainte de Monsieur le General, Guillaume Prendhomme, à propos d'un autre trésorier, ami et amateur de lettres. Enfin, bien que Marot questionne indéniablement le fonctionnement de la déploration funèbre en multipliant les plaintes, le résultat de l'inflexion évangélique de son discours ne constitue guère un renouvellement pérenne du genre et est plutôt une mise en évidence inquiète de ses limites.

Si la Déploration de Florimond Robertet n'est pas une condamnation sans appel de la tradition funèbre des Rhétoriqueurs, c'est parce qu'elle n'est pas, stricto sensu, une déploration funèbre. Le détournement de la canonisation païenne en prêche évangélique porte en effet atteinte à la nature même du discours. Pour comprendre ce point, il convient de qualifier plus précisément les plaintes exprimées par les deux personnifications. L'invective contre la Mort, dans le discours de Republique Françoyse, est d'une telle ampleur, son exécration ("Que mauldit soit ton dard malicieux», v. 218) d’une telle véhémence qu'elle confine à l'accusation (« Ha la meschante, escoutez sa malice», v. 245). L'anaphore «tu as», particulièrement accusatrice, qui commence dès les vers 221-225 déjà cités, rend évident ce glissement du registre démonstratif au genre judiciaire. L’invective contre la Mort déborde donc des limites épidictiques de la rhétorique funèbre traditionnelle pour se transformer en réquisitoire ${ }^{13}$. Cette tension

\footnotetext{
12 Respectivement dans Le thème de la Mort, op. cit., p. 439-483 et La Représentation de la mort dans la littérature de la Renaissance, op. cit., p. 160-193.

${ }^{13}$ Cette accusation n'est pas sans rappeler l'Oraison contemplative devant le crucifix de Barthélémy de Loches, traduite par Marot au début de L'Adolescence clémentine (CEuvres poétiques, op. cit., p. 60-64), dans laquelle la voix poétique accuse Dieu de lui refuser des biens terrestres par malveillance, avant de se rétracter (à partir du vers 134) en rappelant la prudence de ces commandements divins pour écarter le vice et accéder à la rédemption. Elle évoque également, dans un contexte déploratif,
} 
est particulièrement visible dans la dernière strophe, qui ne conclut pas le discours de Republique Françoyse mais au contraire invite à le poursuivre. À cette occasion, elle précise ce qu'elle attend de ses successeurs et donc explicite le registre du discours recherché :

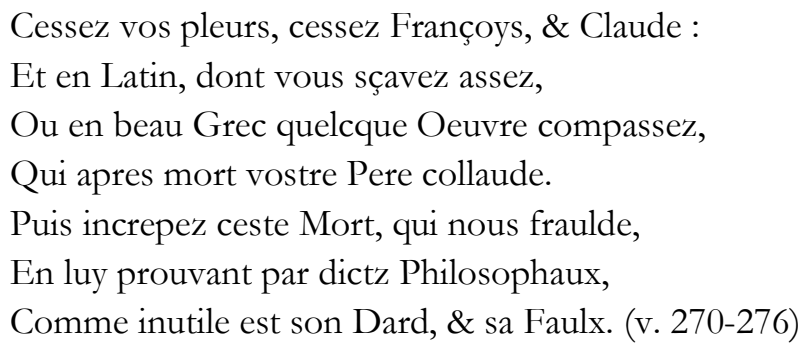

Republique Françoyse invite les deux fils de Robertet non seulement à faire l'éloge du défunt, à le « collaude(r)» (expression du registre épidictique), mais aussi à «prouv(er)» (donc, selon un vocabulaire rhétorique, dans un cadre judiciaire) que la Mort est un méfait, qu'elle « fraulde » autrui.

En s'efforçant de démontrer les torts causés par la Mort et en accusant son inutilité, Republique Françoyse en vient même, paradoxalement, à formuler un début de consolation en indiquant que le défunt accède aux cieux. Christine Martineau-Génieys ${ }^{14}$ remarque qu'elle envisage le Jugement dernier non comme terreur mais Résurrection de la vie :

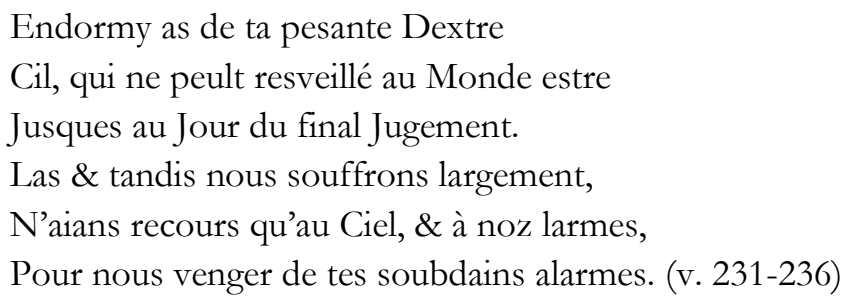

Mais cette consolation chrétienne n'apparaît qu'au détour d'une demi-strophe, entre deux expressions du deuil et de la colère contre la Mort. En voulant faire le mal, la Mort est si inutile qu'elle fait un bien pour le défunt toutefois, et non pour les vivants. Cette dernière se saisit de ce paradoxe et de ce procès d'intention pour formuler une défense reposant sur l'idée que, pour violente que soit son action, elle n'est que bienveillante.

Sa défense est assortie de deux plaintes, dont celle contre l'Église Romaine est à nouveau une véritable accusation. La Mort se défend tout d'abord d'être inutile en avançant que les institutions religieuses mènent un double jeu en condamnant son action tout en tirant profit des cérémonies qu'elle induit : «Peuple seduict [...], / Pourquoy me fais tant de pompes funebres, / Puis que ta bouche inutile me nomme ?» (v. 285-288). La dénonciation de l'inconsistance des hommes évolue rapidement vers celle d'une institution catholique cupide :

Messes sans nombre, \& force Anniversaires,

C'est belle chose, et la façon j'en prise :

Si sont les Chantz, Cloches, et Luminaires :

Mais le mal est en l'avare Prebstrise.

Car si tu n'as vaillant que ta Chemise,

le discours de Peinture, dans La Plainte du Désiré de Lemaire (éd. Dora Yabsley, Paris, Droz, 1932, p. 70-71, v. 65-88) qui s'en prend maladroitement à Dame Nature pour avoir laissé mourir le comte de Ligny (avant également de revenir sur ses propos). Ces emportements font déjà signe vers les tensions inhérentes à toute poésie portant sur la mort d'un proche, mais Marot pousse ici cette contradiction à son terme en transformant la nature même de la plainte épidictique.

${ }^{14}$ Le thème de la Mort, op. cit., p. 447. 
Tiens toy certain, qu'apres le tien trespas

Il n'y aura ne Couvent, ny Esglise,

Qui pour toy sonne, ou chante, ou fasse ung pas. (v. 421-428)

Si la Mort feint de se réjouir de la richesse des pompes funèbres et, en général, de tout rituel catholique fixé par le calendrier, elle met en garde contre l'appât du gain qui gouverne la mise en scène de ces rituels, au moyen d'une rime presque burlesque entre "prebtrise» et "chemise ». La mise en scène excessive de la plainte portant sur un décès, codifiée par les institutions religieuses, vide à nouveau la plainte de sa substance pathétique et la prive de sa capacité à recréer du lien entre les hommes, du sens eschatologique. Blum souligne que ce phénomène normalement religieux et divin qu'est la mort n'est plus que profane et terrestre ${ }^{15}$. Pour Marot, cette incohérence sur le plan de la foi se traduit par une contradiction poétique : l'invective traditionnelle de la Mort depuis le Moyen-Âge, représentée comme humaine et féroce, renvoie à l'amour du monde au détriment d'une foi en Dieu qui devrait convaincre à elle seule que la Mort est un bienfait pour celui qui quitte enfin ses peines terrestres pour rejoindre le Ciel. Ces rituels funèbres, «chant» religieux, mais peut-être aussi celui des poètes de cour payés pour composer en l'honneur du défunt ${ }^{16}$ et consacrer sa gloire, agissent même contre son intérêt, car ils expriment une mort sans au-delà, alors que c'est précisément dans la présence du défunt au Ciel que se trouvent les vraies raisons de se consoler et même de se réjouir.

Pourtant, là encore, ces vers extraits du discours de la Mort font écho à la plainte que le poète formulait déjà en présentant Dame Rommaine dans le cortège funèbre à Torfou. Sa description satirique (v. 58-79), mettant en valeur les possessions de l'Eglise, son bellicisme, son autoritarisme et le vice de ses adeptes, annonce les condamnations par la Mort $^{17}$ de la vénalité et l'esprit de discorde de l'institution papale. L'insistance sur la dimension terrestre de l'Eglise au détriment de considérations spirituelles est la même. Comme pour le discours de Republique Françoyse, cette redondance montre que le poète et la Mort évangélique semblent partager les mêmes vues, mais leurs discours - et en particulier leurs formes respectivement satirique et détournée, ou directe et véhémente - accusent néanmoins un léger décalage. D’un point de vue rhétorique, le glissement de la plainte déplorative à la plainte judiciaire signale que le poème que Marot écrit n'est pas un renouvellement du genre de la déploration funèbre mais seulement une illustration de ses insuffisances portées à leur paroxysme (notamment au moyen de la redondance des discours) jusqu'à changer la nature même du discours. Le poète de cour semble se détourner d'un modèle conventionnel de la déploration hérité des Rhétoriqueurs au profit de la diffusion d'un message évangélique dont l'ampleur et la précision, dans le discours de la Mort, a jusqu’à présent laissé entendre qu’il s'agissait là enfin de la voix de Marot. Pourtant, comme nous le verrons dans notre dernière partie, ce n'est pas sans inquiétude ou tension que le poète transmet et partage ce message formulé par son allégorie.

\section{III- Tension ontologique et eschatologique, entre l'ami et le croyant}

\footnotetext{
${ }^{15}$ La Représentation de la mort dans la littérature de la Renaissance, op. cit., p. 166.

${ }^{16}$ Marot se défend toutefois de ce travers en insistant au début de sa déploration sur la nécessité personnelle qui le pousse à écrire.

17 Cette critique des abus ecclésiastiques et, conjointement, de la papauté, est loin d'être le propre de Marot ou de l'évangélisme. Elle se fonde sur le gallicanisme, courant religieux promouvant l'autonomie, dans le domaine temporel, de l'Église catholique de France par rapport à Rome. Ce courant émerge au tournant des XIII et XIV siècles et connaît un fort regain au début du XVI ${ }^{\mathrm{e}}$ siècle, à l'occasion de la lutte qui opposa Louis XII à Jules II, dans les années 1510-1513, et qui mobilisa poètes et historiographes pour défendre la politique royale contre un pape jugé vicieux, ingrat et belliqueux. Sur cette période, voir la riche étude de Jennifer Britnell, Le Roi très chrétien contre le pape. Écrits antipapaux en français sous le règne de Louis XII, Paris, Classiques Garnier, 2011. Ainsi la description de Marot n'est-elle pas sans rappeler des pièces polémiques de cette période (comme celles de Gringore), parce que les critiques envers la papauté sont toujours aussi virulentes et le seront encore jusqu'à Rabelais au moins (voir la satire des Papimanes aux chapitres XLVIII-LIV du Quart Livre).
} 
La première défense de la Mort face à la charge de Republique Françoyse consiste à se plaindre du fonctionnement hypocrite des institutions supportant son accusation. Mais cette critique est rapidement dépassée par une accusation plus longuement développée de l'inconsistance des hommes en général, qui prétendent croire en un au-delà salvateur et pourtant se plaignent de perdre un être cher. Selon la Mort, il ne faut pas se lamenter mais se réjouir que le défunt accède à « l'eternelle vie » (v. 300), ce qui occulte complètement la relation personnelle ou amicale singulière entre le défunt et l'endeuillé ou le temps nécessaire pour surmonter le deuil. Soit l'interlocuteur est un vrai chrétien, et dans ce cas il n'a pas besoin de cette argumentation, soit il n'est pas un bon croyant, et dans ce cas les arguments de la Mort ne sauront le toucher :

Laisse gemir, et braire les Payens,

Qui n'ont espoir d'eternelle demeure :

Faulte de Foy te donne les moyens

D’ainsi pleurer, quand fault que quelcun meure. (v. 413-416)

La Déploration de Florimond Robertet, qui ressemble à une consolation en ce qu'elle s'efforce de minimiser la douleur liée à la mort, réduit pourtant son argumentation à une tautologie : être croyant, c'est déjà savoir que la mort est une réjouissance. Il n’y a donc aucune perte à consoler, sinon pour des « payens ». Puisque la Mort permet d'accéder au paradis, non seulement elle ne doit pas être blâmée, mais en plus elle doit être désirée. Ainsi, après ses longs développements évangéliques ${ }^{18}$, la Mort résume sa défense et conclut sur son bénéfice :

Mais pour tumber à mon premier propos,

Ne me crains plus, je te pry, ne maulditz:

Car qui vouldra en eternel repos

Avoir de Dieu les promesses, \& dictz,

Qui vouldra veoir les Anges benedictz,

Qui vouldra veoir de son vray Dieu la face,

Brief, qui vouldra vivre au beau Paradis,

Il fault premier que mourir je le fasse. (v. 437-444)

Elle ne nie pas son action, mais répond au procès d'intention qui lui a été fait : son « dard» n'est pas vil ou inutile mais « amoureux » (v. 451), bienveillant. Il est indéniable que tout croyant devrait souhaiter la mort, et pourtant personne ne s'en réjouit... La Mort pointe ainsi une incohérence des hommes, pris entre leur foi (affichée) et leurs passions (même amicales), qui donnent lieu à des plaintes qui, même non ritualisées, n’ont aucune raison d'être. Comment les hommes en général, et Marot en particulier, se positionnent-ils par rapport à cette dénonciation?

Ce point est difficile à déterminer - et cette difficulté est sans doute un premier élément de réponse. Les premiers vers de «l'Autheur» succédant au discours de la Mort laissent apparaitre une critique de ceux qui ne sont pas convertis à ses arguments, qui ne sont pas capables d'écouter sa plainte et de réagir en conséquence :

\footnotetext{
18 Outre les travaux déjà cités de Martineau-Génieys ou Blum, ces arguments ont également été étudiés dans La Poésie religieuse de Clément Marot de Paulette Leblanc (Nizet, 1955), La Religion de Marot de Claude A. Mayer (Nizet, 1960), Marot évangélique de Michael Screech (Genève, Droz, 1967) et, plus récemment, dans Clément Marot: A Renaissance Poet discovers the Gospel. Lutheranism, Fabrism and Calvinism in the Royal Courts of France and of Navarre and in the Ducal Court of Ferrara du même auteur (Leiden, E. J. Brill, 1994) ou Clément Marot and religion : a reassessment in the light of his Psalm paraphrases de Dick Wursten (Leiden, Boston, Brill, 2010).
} 
Quand Mort preschoit ces choses, ou pareilles,

Ceulx qui avoient les plus grandes Oreilles,

N'en desiroient entendre motz quelconques. (v. 453-455)

De même que la Mort formulait une autre plainte après celle de Republique Françoyse, au lieu de la convertir en consolation, de même les «Manans» (v. 459) continuent de se plaindre, comme au tout début d'un poème qui ne semble pas avoir avancé. Marot ne poursuit toutefois pas cette condamnation à l'égard de ces hommes qui n'entendent pas la voix de la foi. Peut-être est-ce parce que cette voix ne s'était pas correctement faite entendre: en effet, dans le concert des arguments évangéliques développés par la Mort pour étayer sa plainte, il est frappant de constater que Robertet disparait complètement $^{19}$, signe que son discours est complètement coupé de son contexte d'énonciation - celle d'un auditoire profondément affecté par la mort d'un être cher. Comme on l'a noté plus haut, sa plainte, même chargée d'arguments solides, ne saurait avoir d'effets. De ce point de vue, et pour reprendre une conclusion de Martineau-Génieys, «le prêche de la Mort dans la Déploration de Florimond Robertet apparaît comme un discours d'initiés à initiés ${ }^{20}$ " plus que comme une plainte adressée à un auditoire présent et appelant une réponse.

Mais peut-on encore parler de plainte lorsque celle-ci manque complètement son but, ne se consacre qu'à l'argumentation de son contenu et ne se soucie même plus d'être entendue ? À défaut de pouvoir répondre avec certitude à cette question, il faut retenir que toutes ces plaintes piétinent, se répètent, parce qu'elles ne parviennent pas à recréer du lien, car les interactions entre plaignant et auditoire reposent sur des malentendus ou s'achèvent par des silences.

Marot lui-même semble mettre à distance la plainte de la Mort, ou du moins ne donne aucun signe de réponse. Alors que dans les premières lignes de la déploration le poète parle à la première personne et en toute transparence ("mon cueur», v. 35 ; «ma plaincte», v. 147), il ne dit rien de sa propre réaction au discours de la Mort. Tandis que tous - «Manans » (v. 459), «Animaux » (v. 479), « Nobles, Prebstres, Marchans » (v. 487) et même le Dieu «Loyre » et les « Naiades» (v. 502-504) - continuent à se lamenter et à craindre la Mort comme dans les premiers vers, le poète semble avoir mystérieusement disparu du cortège qu'il continue pourtant de décrire. On apprend à la toute fin du poème que ce récit n’est plus formulé que de seconde main :

Tel fut conduyt dedans Bloys la Conté

L'ordre funebre, ainsi qu'on m'a compté. (v. 548-549)

Est-ce parce que le poète refuse désormais de s'inclure parmi ceux qui continuent de se plaindre, ou bien ce silence révèle-t-il une autre position, plus nuancée?

Au début de la Déploration de Florimond Robertet, le poète oscillait entre penchant amical et devoir courtisan pour motiver son écriture. Or, la plainte de la Mort, pour évangélique et argumentée qu'elle soit, n'empêche ni les proches de regretter sincèrement l'absence du défunt dans leur vie d'ici-bas, ni les institutions et cérémonies de fonctionner selon leur propres intérêts, plus ou moins corrompus. D’un point de vue courtisan, les arguments évangéliques de la Mort ne consolent en rien le pouvoir de la perte d'un fonctionnaire si compétent - la canonisation glorieuse y parvenait en revanche. La Mort s'adresse donc à de futurs morts en défendant une action qui met fin aux "peines de ce monde » (v. 452), et non aux vivants qui pâtissent de cette perte, encore moins à Republique Françoyse, allégorie

\footnotetext{
19 "L'Autheur », ensuite, n'évoque pas non plus Robertet, absent donc de la deuxième moitié de la déploration qui lui est pourtant consacrée. Cette particularité a également été relevée par Irina Dzero, dans «The Quill Pen in a Funeral Oration: Clément Marot Appropriates the Ancient Genre », (Renaissance and Reformation/Renaissance et Réforme, 2010, vol. 33, n²2, p. 4362), même si elle y voit, conformément à l'esthétique des Rhétoriqueurs, une façon pour Marot de se substituer au mort dans l'accès à la gloire et à la postérité.

${ }^{20}$ Le thème de la Mort, op. cit., p. 475.
} 
du pouvoir, qui pleure la perte d'un tel atout politique. Peut-être est-ce la raison pour laquelle l'auteur se tait après le discours de la Mort et laisse au lecteur le temps de trouver en lui la force ou l'humilité de se laisser consoler. Dans la Déploration de Florimond Robertet, le lecteur n'est que renvoyé à sa foi. La démonstration de l'incohérence du deuil pour tout croyant est une invitation à prendre en compte la contradiction et la faiblesse inhérente à l'expérience humaine, dont il ne s'agit pas de nier les affects qui la touchent immanquablement. La Déploration de Florimond Robertet est non seulement - et volontairement - en décalage par rapport à sa motivation courtisane, mais aussi par rapport à sa vocation rhétorique, puisqu'il s'agit moins de persuader que peut-être - et cela est plus évident dans les poèmes funèbres de Marguerite de Navarre tels que le Dialogue en forme de vision nocturne et La Navire ${ }^{21}$ de ménager un espace pour la foi où la grâce pourra agir. En d'autres termes, cette plainte évangélique de la Mort n'appelle peut-être ni consolation ni nouvelle plainte, mais précisément le silence de l'attente de la grâce. Avec la Mort évangélique de Marot, on ne peut plus faire rimer « dueil » et « larmes d'œil » (v. 406-408), on ne peut plus se lamenter sur le corps d'un illustre défunt comme le ferait n'importe quel courtisan. Face à cet événement, le cœur - siège de l'amour et de la foi - est engagé. Mais cela bouleverse la portée de la plainte et de la consolation, ainsi que les liens qu'elles s'efforcent de restaurer : enjeux que la Déploration de Florimond Robertet montre sans les résoudre.

Contrairement à des lectures plus univoques et subversives de la Déploration de Florimond Robertet comme rejet de la Grande Rhétorique et manifeste évangélique, une étude attentive aux plaintes qu'elle met en scène, en particulier à leur multiplication qui tend au piétinement, permet d'envisager ce poème sous un jour plus incertain, en tant qu'élément d'une réflexion de Marot sur la foi, confrontée à des réalités sociales et humaines qui en questionnent le sens. La caractérisation précise des plaintes (au moyen d'une typologie qui emprunte à la rhétorique son vocabulaire et ses outils d'analyses) permet de dévoiler des glissements, des boitements du message qui, loin de systématiser ou simplifier l'analyse littéraire, nuancent et enrichissent la compréhension de cette déploration aux confins des genres et des pratiques. La reformulation constante de plaintes au cours du poème souligne à la fois le rôle crucial de ce discours - créer du lien et du sens entre les hommes, alors qu'ils sont confrontés à l'épreuve particulièrement destructrice qu'est le deuil, en réaffirmant des valeurs éthiques, sociales ou religieuses mais aussi ses limites - la plainte seule ne suffit pas à faire le consensus autour de ces valeurs. Le pathos doit être assorti d'une écoute et d'une réponse raisonnée (même si la plus raisonnée est peut-être le silence) pour avoir du sens, ériger sa cause en valeurs susceptibles de recréer du lien entre les interlocuteurs. Si elle ne s'en tient qu'à une expression solitaire de la douleur, la plainte glisse vers la complaisance ou sombre dans une vaine révolte. Morceau de bravoure rhétorique, manifeste évangélique inquiet, la Déploration de Florimond Robertet est donc aussi un appel à écouter les plaintes, non seulement pour y apporter la réponse adéquate, mais aussi parce qu'en elles gisent les plus profondes contradictions - et richesses - de l'homme privé et de l'homme social.

Ellen Delvallée

Université Grenoble-Alpes / Rutgers University

\footnotetext{
${ }^{21}$ Déjà, dans le récit de la résurrection de Lazare ( $n$ n 11), alors même que Jésus se montre confiant dans sa capacité à faire revivre son ami, il se sent troublé intérieurement jusqu'aux larmes devant son tombeau. Après Marot, Marguerite de Navarre, dans La Navire consacrée à la mort de son frère François I ${ }^{\text {er }}$, met en scène cette contradiction entre tristesse humaine et foi à travers un dialogue entre elle-même, effondrée de douleur, et son frère, qui lui apparait en songe et lui rappelle combien elle devrait être heureuse pour lui. Au terme de ce dialogue où, comme dans la déploration de Marot, aucun des interlocuteurs ne parvient à convaincre l'autre, la prière intime apparaît comme le seul moyen de sortir de cette contradiction.
} 